\title{
Tetraalkylammonium Picrates in the Dichloromethane-Water System: Ion-Pair Formation and Liquid-Liquid Distribution of Free Ions and Ion Pairs
}

\author{
Shoichi Katsuta · Hirokazu Wakabayashi · \\ Mariko Tamaru • Yoshihiro Kudo · Yasuyuki Takeda
}

Published online: 9 May 2007

(C) Springer Science+Business Media, LLC 2007

\section{Erratum to: Int J Solution Chem}

DOI 10.1007/s10953-007-9121-x

Listed are corrections to the above article:

1. Table 3, fourth column, heading

$$
\begin{aligned}
& \log _{10} K_{\mathrm{D}}^{\mathrm{o}}\left(\mathrm{C}^{+} \mathrm{A}^{-}\right) \\
& \log _{10} K_{\mathrm{D}}^{\mathrm{o}}\left(\mathrm{C}^{+} \mathrm{A}^{-}\right) . \\
& -\frac{E_{\mathrm{o}, \mathrm{CA}}-E_{\mathrm{o}, \mathrm{CA}}}{2.303 R T} . \\
& -\frac{E_{\mathrm{o}, \mathrm{CA}}-E_{\mathrm{w}, \mathrm{CA}}}{2.303 R T} \\
& K_{I P, \mathrm{o}} \\
& K_{\mathrm{IP}, \mathrm{o}} \\
& K_{I P, \mathrm{w}} \\
& K_{\mathrm{IP}, \mathrm{w}}
\end{aligned}
$$$$
\text { should read }
$$

The online version of the original article can be found at http://dx.doi.org/10.1007/s10953-007-9121-x

S. Katsuta $(\varangle) \cdot$ H. Wakabayashi · M. Tamaru · Y. Kudo · Y. Takeda Department of Chemistry, Faculty of Science, Chiba University, 1-33 Yayoi-cho, Inage-ku, Chiba 263-8522, Japan

e-mail: katsuta@faculty.chiba-u.jp 